Journal of Fundamental and Applied Sciences

ISSN 1112-9867

Available online at

http://www.jfas.info

\title{
A LIXISOL MICROBIAL ACTIVITY UNDER URBAN WASTES COMPOSTS EFFECT IN DONSIN, BURKINA FASO
}

\author{
D. Bambara*, A. Thiombiano, V. Hien
}

Institut de l'Environnement et de Recherches Agricoles (INERA) - 01 BP 476 Ouagadougou 01 Burkina Faso

Received: 14 September 2014 / Accepted: 6 October 2014 / Published online: 31 December 2014

\begin{abstract}
Mineralization causes soil carbon loss. This study focuses soil microbial activity measurement in relation with the application of six types of composts in sorghum three-year experiment. Composts were applied single or combined with urea. Organic carbon content, microbial biomass, respirometry and carbon global mineralization rate were evaluated. In comparison to the control, single composts treatments parameters variation rates ranged from -1 to $+71 \%$. When composts were combined with urea, parameters variations rates ranged from -8 to $+107 \%$. Under single urea, these parameters variation rates ranged from $-14 \%$ to $+31 \%$. Best composts seemed to be those produced with various and balanced composting materials. Ecofriendly composts adoption could improve soil organic carbon content for sustainable microbial process.
\end{abstract}

Keywords: Agro systems; carbon; respirometry; Burkina Faso.

\section{INTRODUCTION}

Depuis fort longtemps, la matière organique des sols (MOS) a été perçue comme le facteur essentiel de la fertilité des sols cultivés[1-3]. Plus récemment, des fonctions environnementales de premier plan sont attribuées aux MOS car elles peuvent contribuer à la lutte contre l'effet de serre additionnel en stockant du carbone dans les sols[4,5].

Author Correspondence, e-mail: das.bambara@coraf.org

ICID: 1124399 
Selon[6] la masse de carbone stocké dans le sol au niveau mondial est estimée à 2000 et 2 $500 \mathrm{Gt}$ dont 27 à $36 \%$ dans les zones sèches.

Le sol est alors considéré comme le plus grand réservoir superficiel de carbone[7]. Dans les zones sèches, la dégradation des sols est essentiellement caractérisée par de faibles taux de matière organique[8-10]. Au Burkina Faso, 38\% des sols sont affectés par les processus de dégradation induits par les changements climatiques et la mauvaise gestion anthropique[11] et 5 à $40 \%$ des terres du Plateau Central sont soumises à une dégradation sévère[12]. Face à cette dégradation et la réduction des rendements des cultures qui en découle, les producteurs utilisent la fumure organique comme un moyen de restauration de la fonction productive des sols[1315].

L'apport au sol de fumure organique telle que le compost améliore en particulier la teneur en carbone du sol et stimule l'activité microbienne[10, 16-19]. Mais la variation du stock de carbone organique dans les sols est la résultante du bilan entre les flux entrants (apports organiques notamment) et les flux sortants (minéralisation microbienne du carbone du sol)[5]. De ce fait, l'évolution des stocks de carbone dans le sol dépend de l'intensité de l'activité microbienne. Une diminution de la teneur en carbone organique obtenue par une augmentation de la vitesse de minéralisation, peut mettre en jeu des quantités de carbone très importantes par rapport aux flux nets annuels d'échange avec l'atmosphère[5]. Au Burkina Faso, les travaux sur l'influence des apports organiques notamment les composts de déchets urbains sur l'activité microbienne du sol sont rares. Pourtant, dans le double contexte de manque de substrat organique compostable particulièrement en zone périurbaine (pour des raisons d'usage domestique et de vente) et d'augmentation considérable de la production de déchets urbains liés à l'urbanisation croissante en zone urbaine[20], la valorisation agricole des déchets urbains après compostage en zone périurbaine peut être envisagée. En effet, la ville de Ouagadougou en a produit 300000 t en 2007 et en 2025, 1000000 de tonnes de déchets sont attendus[21].

L'étude visait à évaluer, à partir d'expérimentation en milieu réel, l'impact de six formules de composts de déchets urbains de Ouagadougou sur la teneur en carbone du sol et l'activité microbienne (à travers la biomasse microbienne et la respiration du sol) et à identifier la formule de compost qui améliore le mieux la teneur en carbone du sol.

\section{MATÉRIEL ET MÉTHODES}

\subsection{Milieu d'étude}

L'étude a été conduite à Donsin, localisé à $12^{\circ} 35^{\prime} 01^{\prime \prime}$ de latitude Nord et $01^{\circ} 24^{\prime} 06^{\prime \prime}$ ' de longitude Ouest. Avec une superficie de $9,38 \mathrm{~km}^{2}$, il est situé à $25 \mathrm{~km}$ au Nord-est de 
Ouagadougou, chef-lieu de la région du Centre. Inclus dans l'espace géographique du GrandOuaga[22], Donsin est une zone semi-rurale où les composts de déchets urbains de Ouagadougou peuvent être valorisés. Sa population compte environ 1889 habitants avec une densité de 201 habitants/ $\mathrm{km}^{2}$ [23]. Les activités socioéconomiques comprennent l'agriculture pluviale (sorgho dominant), le maraîchage, l'élevage, la pêche, le petit commerce et l'artisanat. Donsin appartient au secteur phytogéographique nord-soudanien[24] dans la zone climatique sahélo-soudanienne avec une pluviométrie annuelle variant entre 700 et $900 \mathrm{~mm}$, figure 1. Les différents types de sols rencontrés appartiennent à la classe des sols ferrugineux tropicaux[25]. La végétation est constituée de savanes arbustives à arborées. Le contexte écologique de Donsin est marqué par des sols et un couvert végétal dégradés. La dégradation des sols se caractérise par leur faible niveau de fertilité lié notamment à leurs faibles teneurs en matière organique [10], généralement en dessous de 1\%[9].

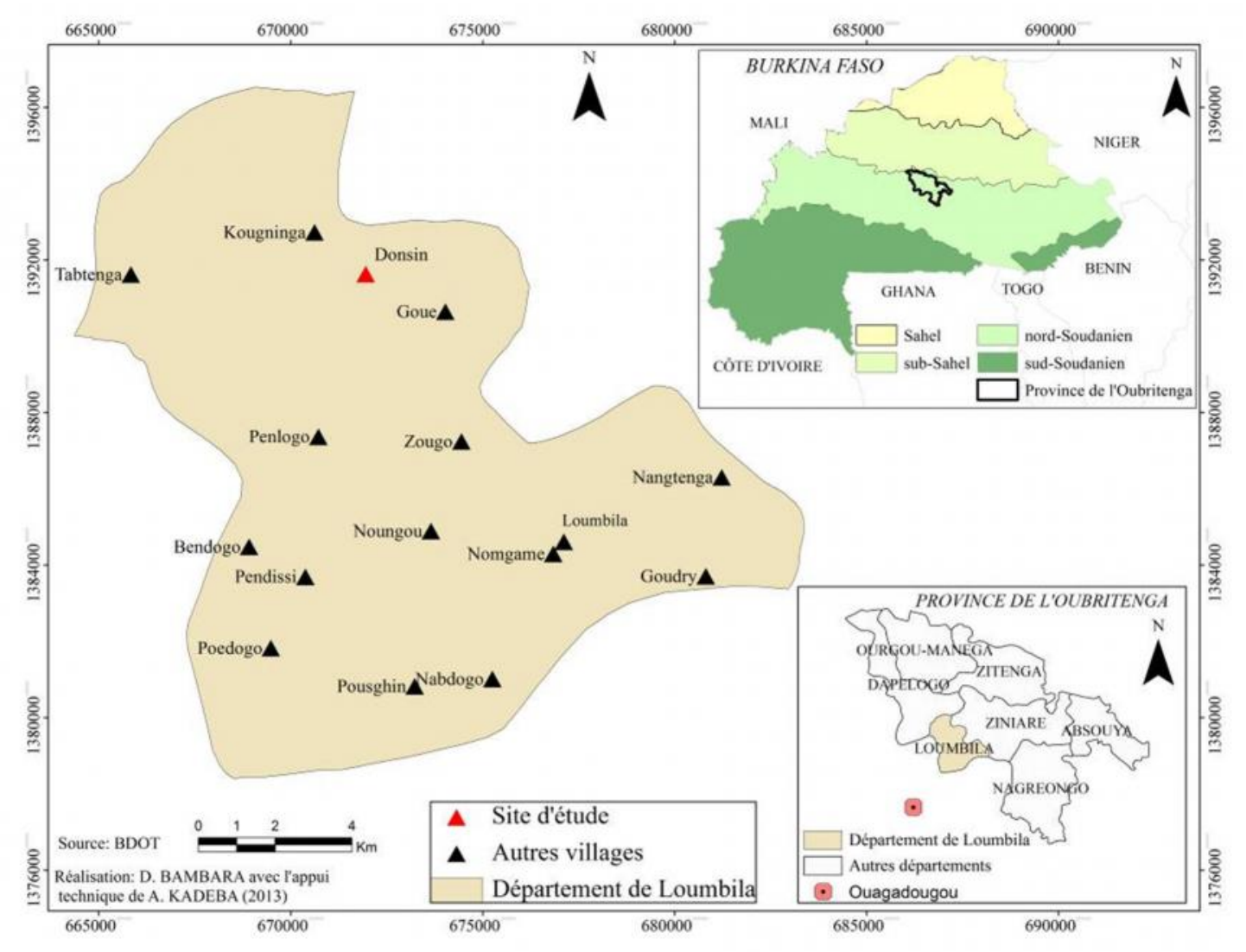

Fig.1. Situation géographique du village de Donsin 


\subsection{Choix et caractérisation du site expérimental}

L'essai a été réalisé en milieu réel. L'étude pédologique réalisée à partir de trois profils indique que le sol est un lixisol endopetroplinthique[26], équivalant dans la classification française, à un sol ferrugineux tropical lessivé induré moyennement profond[27]. Les caractéristiques pédologiques de l'horizon superficiel 0-20 cm sont reportées dans le tableau 1.

Tableau 1. Caractéristiques pédologiques de l'horizon de surface $(0-20 \mathrm{~cm})$ du sol du site expérimental (Moyennes \pm Ecart type, $\mathrm{n}=3$ profils)

\begin{tabular}{|c|c|}
\hline Propriétés du sol & Moyennes \pm Ecart type \\
\hline \multicolumn{2}{|l|}{ Granulométrie (\%) } \\
\hline Argile & $15,77 \pm 2,50$ \\
\hline Limons totaux & $36,40 \pm 5,24$ \\
\hline Sables totaux & $47,83 \pm 6,42$ \\
\hline Texture & (Limoneuse) \\
\hline Type de sol (WRB, 2006) & (Lixisol endopétroplinthique) \\
\hline Matière organique $(\%)$ & $0,744 \pm 0,150$ \\
\hline Carbone total $(\%)$ & $0,432 \pm 0,087$ \\
\hline Azote total $(\%)$ & $0,033 \pm 0,004$ \\
\hline $\mathrm{C} / \mathrm{N}$ & $13,31 \pm 3,49$ \\
\hline Phosphore total (ppm) & $137,62 \pm 12,82$ \\
\hline Phosphore assimilable (ppm) & $3,62 \pm 1,16$ \\
\hline Potassium total (ppm) & $1725,45 \pm 387,62$ \\
\hline $\mathrm{pH}$ eau & $5,8 \pm 0,3$ \\
\hline
\end{tabular}

Les sables totaux constituent la principale classe granulométrique et la texture est limoneuse[28]. Se référant aux normes rapportées par[29], l'horizon de surface du sol possède des teneurs, basse en matière organique, en azote et en phosphore totaux, moyenne en potassium total, très basse en phosphore assimilable. Cet horizon est moyennement acide, tableau 1.

\subsection{Composts}

Les composts utilisés ont été produits à partir du mélange de déchets solides urbains biodégradables. Le compost 1 était produit à partir du mélange de $60 \%$ de déchets d'abattoir $(\mathrm{DA}=$ contenu des panses de ruminants $)+40 \%$ de déchets verts $(\mathrm{DV}=$ Feuilles de Kaya 
senegalensis, Cassia seamea, Eucalyptus camaldulensis), le compost $2: 40 \%$ de DA + 20\% de déchets de cuisine $(\mathrm{DC}=$ Epluchures diverses, restes de fruits divers, légumes divers abîmés, restes de repas divers) $+40 \%$ de DV, le compost $3: 30 \%$ de DA $+30 \%$ de DC $+40 \%$ de DV, le compost $4: 20 \%$ de DA $+40 \%$ de DC $+40 \%$ de DV, le compost $5: 60 \%$ de DC $+40 \%$ de DV et le compost $6: 100 \%$ de DV. Les principales propriétés des composts sont consignées dans le tableau 2 . 
Tableau 2. Propriétés chimiques des composts (moyennes \pm écart type, $\mathrm{n}=3$ prélèvements)

\begin{tabular}{lllllll}
\hline Paramètres & Compost 1 & Compost 2 & Compost 3 & Compost 4 & Compost 5 & Compost 6 \\
\hline $\mathrm{MS}(\%)$ & $60,93 \mathrm{~b} \pm 4,29$ & $68,50 \mathrm{a} \pm 8,52$ & $67,00 \mathrm{ab} \pm 9,30$ & $69,39 \mathrm{a} \pm 3,69$ & $72,05 \mathrm{a} \pm 6,84$ & $45,87 \mathrm{c} \pm 6,90$ \\
$\mathrm{Ct}\left(\mathrm{g} \mathrm{kg}^{-1}\right)$ & $363,85 \mathrm{~cd} \pm 9,60$ & $391,1 \mathrm{~b} \pm 20,1$ & $366,75 \mathrm{c} \pm 7,30$ & $335,10 \mathrm{e} \pm 10,10$ & $343,90 \mathrm{de} \pm 12,90$ & $415,10 \mathrm{a} \pm 7,10$ \\
$\mathrm{Nt}\left(\mathrm{g} \mathrm{kg}^{-1}\right)$ & $21,80 \mathrm{ab} \pm 9,70$ & $25,80 \mathrm{a} \pm 0,60$ & $26 \mathrm{a} \pm 3,80$ & $21,50 \mathrm{ab} \pm 5,80$ & $21,10 \mathrm{ab} \pm 1,00$ & $16,10 \mathrm{~b} \pm 3,30$ \\
$\mathrm{C} / \mathrm{N}$ & $16,69 \mathrm{ab} \pm 8,63$ & $15,17 \mathrm{~b} \pm 1,08$ & $14,28 \mathrm{~b} \pm 1,75$ & $15,50 \mathrm{~b} \pm 5,10$ & $16,34 \mathrm{~b} \pm 1,41$ & $25,78 \mathrm{a} \pm 6,24$ \\
$\mathrm{Pt}\left(\mathrm{g} \mathrm{kg}^{-1}\right)$ & $1,85 \mathrm{a} \pm 0,37$ & $1,73 \mathrm{a} \pm 0,11$ & $1,83 \mathrm{a} \pm 0,06$ & $1,98 \mathrm{a} \pm 0,33$ & $1,81 \mathrm{a} \pm 0,18$ & $0,89 \mathrm{~b} \pm 0,17$ \\
$\mathrm{Kt}\left(\mathrm{g} \mathrm{kg}^{-1}\right)$ & $5,86 \mathrm{c} \pm 0,54$ & $7,16 \mathrm{c} \pm 0,29$ & $13,68 \mathrm{a} \pm 2,41$ & $7,16 \mathrm{c} \pm 0,72$ & $10,09 \mathrm{~b} \pm 1,07$ & $7,82 \mathrm{c} \pm 0,90$ \\
$\mathrm{Ca}\left(\mathrm{g} \mathrm{kg}^{-1}\right)$ & $19,22 \mathrm{~d} \pm 0,86$ & $22,53 \mathrm{c} \pm 3,07$ & $28,12 \mathrm{~b} \pm 2,26$ & $35,39 \mathrm{a} \pm 0,64$ & $33,53 \mathrm{a} \pm 0,51$ & $27,92 \mathrm{~b} \pm 1,27$ \\
$\mathrm{Mg}\left(\mathrm{g} \mathrm{kg}^{-1}\right)$ & $2,12 \mathrm{c} \pm 0,10$ & $2,62 \mathrm{~b} \pm 0,46$ & $2,95 \mathrm{ab} \pm 0,15$ & $2,66 \mathrm{~b} \pm 0,24$ & $3,13 \mathrm{a} \pm 0,18$ & $3,01 \mathrm{ab} \pm 0,26$ \\
$\mathrm{pH} \mathrm{eau}$ & $8,6 \mathrm{ab} \pm 0,4$ & $8,8 \mathrm{a} \pm 0,2$ & $8,1 \mathrm{~b} \pm 0,2$ & $8,4 \mathrm{ab} \pm 0,3$ & $8,5 \mathrm{ab} \pm 0,4$ & $8,3 \mathrm{ab} \pm 0,3$
\end{tabular}

La matière sèche $(M S)$ est exprimée en \% du produit frais; ;es autres analyses sont exprimées par rapport au poids sec des composts. Ct : Carbone total ; Nt : Azote total ; Pt : Phosphore total ; Kt: Potassium total, Ca :Calcium ; Mg : Magnésium.

Les moyennes sur la même ligne qui ont la même lettre ne diffèrent pas significativement au seuil de 5 \% (Test de Fischer, LSD) 
Les teneurs en éléments chimiques étaient variables d'un compost à l'autre mais conformes aux critères de la norme NF U 44-05[30]. La teneur en azote total est basse. Les rapports $\mathrm{C} / \mathrm{N}$ sont élevés indiquant que les composts sont des amendements organiques[31]. Les teneurs en phosphore total sont basses. Le pH est moyennement alcalin pour les composts 3, 6 et 4 et fortement alcalin pour les composts 5,1 et 2 .

\subsection{Matériel végétal}

La variété de sorgho, Sorghum bicolor (L.) Moench, CEF 382/2-1-1 (Sariaso 11) a été utilisée. C'est une variété sélectionnée assez résistante à la sécheresse (adaptée à la zone comprise entre les isohyètes 400 et $700 \mathrm{~mm}$ ) et qui valorise bien la fumure organique. Son cycle est de 100 jours [32].

\subsection{Dispositif expérimental et modalités comparées}

Le dispositif expérimental était constitué de 4 blocs de Fischer (36 m x 13 m chacun) comportant 14 traitements (tableau 3) correspondant au total à 56 parcelles élémentaires ( $5 \mathrm{~m} \mathrm{x} 4 \mathrm{~m}$ ). Les allées entre les parcelles consécutives étaient de $1 \mathrm{~m}$. Les blocs étaient dispersés mais les distances qui les séparaient étaient comprises entre 10 et $30 \mathrm{~m}$.

Tableau 3. Liste des traitements expérimentaux

\begin{tabular}{|l|l|l|}
\hline Numéros d'ordre des traitements & Désignations & Abréviations \\
\hline 1 & Compost 1 & C1 \\
2 & Compost 2 & C2 \\
3 & Compost 3 & C3 \\
4 & Compost 4 & C4 \\
5 & Compost 5 & C5 \\
6 & Compost 6 & C6 \\
7 & Compost 1 + Urée & C1N \\
8 & Compost 2 + Urée & C2N \\
9 & Compost 3 + Urée & C3N \\
10 & Compost 4 + Urée & C4N \\
11 & Compost 5 + Urée & C5N \\
12 & Compost 6 + Urée & C6N \\
13 & Urée & N \\
14 & Témoin absolu sans apport de & TA \\
\hline
\end{tabular}


La prise en compte des traitements 7 à 12 se justifie par les faibles valeurs azotées des composts et le rôle de premier rang que joue cet élément dans le développement des cultures[33].

\subsection{Déroulement de l'essai et procédure d'échantillonnage du sol}

L'essai a été conduit sur la même parcelle en 2010, 2011, 2012. L'apport des fertilisants a été réalisé à la levée des plants de sorgho, localisé au pied du plant à la dose de $3 \mathrm{t} \mathrm{MS} \mathrm{ha-1} \mathrm{an}^{-1}$ pour les composts et $50 \mathrm{~kg} \mathrm{ha}^{-1} \mathrm{an}^{-1}$ pour l'urée. Le sorgho a été semé à la densité de $0,8 \mathrm{~m} \mathrm{x} 0,4 \mathrm{~m}$ et démarié à trois plants par poquet une semaine après le semis. Les semis ont été effectués dans la première quinzaine de juillet de chaque campagne agricole. Les récoltes ont été effectuées en novembre de chaque année. A la fin des 3 ans d'essai, une analyse physico-chimique d'échantillons de sol a été réalisée. Sur chaque parcelle (traitement), les prélèvements de sols ont été réalisés dans l'horizon 0-20 cm[34]. L'échantillonnage du sol, réalisé après la dernière récolte du sorgho en novembre 2012, a été fait à la tarière dans les interlignes centrales de semis à $20 \mathrm{~cm}$ des poquets. Huit prélèvements ont été effectués à l'intérieur de chaque parcelle. Ces prélèvements sont incorporés dans un seau plastique, puis mélangés plusieurs fois de façon à les rendre homogènes et $1 \mathrm{~kg}$ de sol est prélevé pour les analyses de laboratoire. Une partie a été conditionnée et conservée au frais pour les analyses biologiques et une autre partie a été séchée pour la détermination du carbone organique et de la terre fine. Cinquante six échantillons composites[35] ont été ainsi constitués pour les différentes analyses.

\subsection{Procédure de mesure des variables}

\subsubsection{Carbone organique}

Il a été déterminé au moyen d'un auto-analyseur élémentaire par oxydation de l'échantillon de sol puis par réduction des gaz de combustion. La séparation s'effectue sur colonne chromatographique[36].

\subsubsection{Terre fine}

La terre fine est la fraction de terre qui reste lorsqu'on retire les éléments grossiers (> $2 \mathrm{~mm}$ au tamis) [37]. La terre fine a été déterminée sur un échantillon de sol sec, après avoir séparé la fraction grossière de l'échantillon, Poids terre fine = (poids échantillon - poids éléments grossiers). Le taux de terre fine (en \%) est obtenu par la formule :

$\mathrm{Tf}(\%)=($ Poids terre fine / poids échantillon $) \times 100$. 


\subsubsection{Biomasse microbienne}

La méthode fumigation-extraction[38] a été adoptée car appropriée pour les sols pauvres en matière organique[39]. Le carbone de la biomasse microbienne est dosé à partir d'échantillons de $100 \mathrm{~g}$ de sol fumigés $(\mathrm{F})$ et $100 \mathrm{~g}$ de sol non fumigés (nF). Cette méthode consiste à traiter le sol à fumiger avec de l'éthanol libre chloroforme $\left(\mathrm{CHCl}_{3}\right)$ pendant $24 \mathrm{~h}$. Ce procédé tue la presque totalité des microorganismes du sol dont le contenu cellulaire est minéralisé et se dégage sous forme de $\mathrm{CO}_{2}$. Après avoir soustrait le fumigant par des évacuations répétées, ces échantillons sont incubés à $30^{\circ} \mathrm{C}$ avec les échantillons de la fraction non fumigée en présence de $\mathrm{NaOH}(0,5 \mathrm{~N})$ qui piège le $\mathrm{CO}_{2}$ dégagé des sols[40]. Au terme de la durée d'incubation, les sols fumigés et les témoins ont été prélevés après 7 et $14 \mathrm{j}$, en vue du dosage du $\mathrm{CO}_{2}$ par titration avec du $\mathrm{HCl} 0,1 \mathrm{~N}$. La quantité de carbone de la biomasse microbienne (CBM) est obtenue par la formule : $\mathrm{CBM}=[\mathrm{F}$ $(0-7)-\mathrm{nF}(7-14)] / \mathrm{kc}$

Où : kc est un facteur de correction représentant la fraction minéralisable en $\mathrm{CO}_{2}$ du carbone de la biomasse, il s'élève à $0,41[41] ; \mathrm{F}(0-7)$ représente le $\mathrm{C}-\mathrm{CO}_{2}$ dégagé par l'échantillon de sol fumigé entre 0-7 j et nF (7-14), le C-CO $\mathrm{CO}_{2}$ dégagé par l'échantillon de sol non fumigé entre 7-14 j.

\subsubsection{Respiration du sol (minéralisation du carbone)}

La méthode la plus ancienne et la plus simple pour évaluer l'activité microbienne du sol consiste

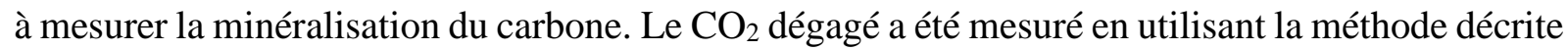
par[42]. Les échantillons de sol, maintenus à une teneur en eau proche de la capacité au champ sont incubés à $28^{\circ} \mathrm{C}$ afin de permettre la reprise d'activité des microorganismes[43-44]. L'incubation a duré $21 \mathrm{j}[45]$. Les mesures ont été effectuées quotidiennement pendant une semaine puis tous les deux jours jusqu'au $21^{\mathrm{e}} \mathrm{j}$. La procédure est celle décrite pour la biomasse microbienne. Le taux de minéralisation globale du carbone (TMG) a été calculé selon la formule : $\mathrm{TMG}=\mathrm{C}-\mathrm{CO} 2 / \mathrm{C}$ org. $\mathrm{x} 100[46]$.

\subsection{Analyses statistiques}

Les données collectées ont été soumises à une analyse de variance (ANOVA) à un facteur en utilisant le test des moindres carrés de Fischer (LSD) pour comparer les moyennes des différents paramètres sous divers composts. Le seuil de significativité a été défini à $\mathrm{P}<0,05$. Les comparaisons ont été faites selon trois niveaux : (i) les différents traitements de composts seuls avec le témoin, (ii) les différents traitements de composts couplés à l'urée avec le témoin et le traitement à base d'urée, (iii) les différents traitements de composts seuls avec ceux de composts 
couplés à l'urée. La corrélation de Pearson a été utilisée pour établir les relations entre les variables au seuil de 5\%. Une modélisation des données par régression linéaire a été établie entre les variables pour lesquelles la corrélation de Pearson était statistiquement significative en vue de déterminer les équations des modèles. Le logiciel XLSTAT-Pro 7.5.2[47] a été utilisé pour ces analyses. Le tracé des graphiques a été réalisé avec le même logiciel.

\section{RÉSULTATS}

3.1. Variabilité du carbone organique ( $C$ org), du taux de terre fine, du carbone de la biomasse microbienne (CBM), de la respiration et du taux de minéralisation globale du carbone (TMG)

Les composts ont influencé différemment la teneur en carbone organique, la terre fine, la biomasse microbienne, la respiration et le taux de minéralisation globale du sol comparativement au témoin sans fertilisation (tableau 4). Les taux de variation des paramètres par rapport au témoin fluctuaient entre -1 et $+37 \%$ pour $\mathrm{C}$ org,+15 et $+29 \%$ pour la terre fine, +27 et $+71 \%$ pour $\mathrm{CBM}, 0$ et $+53 \%$ pour la respiration, +4 et $+63 \%$ pour TMG. Quand les composts sont couplés à l'urée les variations comparativement au témoin fluctuaient entre +10 et $+40 \%$ pour $\mathrm{C}$ org, +22 et $+33 \%$ pour la terre fine, +39 et $+107 \%$ pour CBM, +3 et $+68 \%$ pour la respiration, -8 et $+75 \%$ pour TMG. Les variations de $\mathrm{C}$ org, de terre fine, de CBM, de la respiration et du TMG des traitements à base d'urée comparativement au témoin étaient respectivement $+15 \%,-1 \%,+31 \%,+4 \%$ et $-14 \%$. Les traitements de composts couplés à l'urée ont présenté des dynamiques plus importantes comparativement aux traitements à base de composts seuls, toutefois, les différences entre les traitements n'étaient pas significatives ( $p>0,05)$ (Figure 2. ABCD). 
Tableau 4. Variabilité du carbone organique (C org), du taux de terre fine, du Carbone de la Biomasse Microbienne (CBM), de la respiration $\left(\mathrm{C}-\mathrm{CO}_{2}\right)$ et du taux de minéralisation globale $(\mathrm{TMG})$ du carbone du sol sous composts seuls et sous composts couplés avec

l'urée (Moyennes \pm écart type)

\begin{tabular}{llllll}
\hline Traitements & C org $\left(\mathrm{mg} \mathrm{kg}^{-1}\right)$ & Terre fine $(\%)$ & CBM $\left(\mathrm{mg} \mathrm{kg}^{-1}\right)$ & $\mathrm{C}-\mathrm{CO}_{2}\left(\mathrm{mg} \mathrm{kg}^{-1}\right)$ & TMG $(\%)$ \\
\hline C1 & $4438 \mathrm{a} \pm 925$ & $69,48 \mathrm{a} \pm 3,56$ & $53,37 \mathrm{ab} \pm 15,37$ & $142,08 \mathrm{a} \pm 51,43$ & $3,14 \mathrm{a} \pm 1,02$ \\
$\mathrm{C} 2$ & $5083 \mathrm{a} \pm 1166$ & $64,77 \mathrm{a} \pm 8,58$ & $50,96 \mathrm{ab} \pm 7,81$ & $109,98 \mathrm{a} \pm 48,40$ & $2,19 \mathrm{a} \pm 0,37$ \\
$\mathrm{C} 3$ & $5748 \mathrm{a} \pm 1644$ & $70,11 \mathrm{a} \pm 2,57$ & $65,094 \mathrm{a} \pm 12,64$ & $112,55 \mathrm{a} \pm 37,85$ & $2,00 \mathrm{a} \pm 0,83$ \\
$\mathrm{C} 4$ & $67,36 \mathrm{a} \pm 9,72$ & $50,06 \mathrm{ab} \pm 12,53$ & $126,90 \mathrm{a} \pm 49,64$ & $2,74 \mathrm{a} \pm 1,26$ \\
$\mathrm{C} 5$ & $4425 \mathrm{a} \pm 1293$ & $62,10 \mathrm{ab} \pm 3,58$ & $48,26 \mathrm{ab} \pm 12,79$ & $115,88 \mathrm{a} \pm 45,25$ & $2,10 \mathrm{a} \pm 0,56$ \\
$\mathrm{C} 6$ & $4395 \mathrm{a} \pm 859$ & $65,78 \mathrm{a} \pm 4,09$ & $48,86 \mathrm{ab} \pm 14,10$ & $92,72 \mathrm{a} \pm 21,22$ & $2,25 \mathrm{a} \pm 1,24$ \\
TA & $4155 \mathrm{a} \pm 1504$ & $54,21 \mathrm{~b} \pm 2,54$ & $38,03 \mathrm{~b} \pm 10,14$ & $92,96 \mathrm{a} \pm 39,06$ & $1,93 \mathrm{a} \pm 0,53$ \\
\hline C1N & $4205 \mathrm{a} \pm 1434$ & $72,25 \mathrm{a} \pm 2,87$ & $54,61 \mathrm{~b} \pm 15,69$ & $156,54 \mathrm{a} \pm 57,12$ & $3,38 \mathrm{a} \pm 1,52$ \\
$\mathrm{C} 2 \mathrm{~N}$ & $5500 \mathrm{a} \pm 1582$ & $68,75 \mathrm{a} \pm 8,57$ & $55,83 \mathrm{ab} \pm 8,43$ & $116,32 \mathrm{a} \pm 40,41$ & $2,27 \mathrm{a} \pm 1,15$ \\
$\mathrm{C} 3 \mathrm{~N}$ & $5875 \mathrm{a} \pm 1159$ & $71,93 \mathrm{a} \pm 3,22$ & $78,90 \mathrm{a} \pm 15,65$ & $130,98 \mathrm{a} \pm 45,10$ & $2,65 \mathrm{a} \pm 0,59$ \\
$\mathrm{C} 4 \mathrm{~N}$ & $5308 \mathrm{a} \pm 1126$ & $70,46 \mathrm{a} \pm 12,21$ & $54,05 \mathrm{~b} \pm 12,14$ & $134,79 \mathrm{a} \pm 64,93$ & $2,69 \mathrm{a} \pm 1,41$ \\
$\mathrm{C} 5 \mathrm{~N}$ & $4893 \mathrm{a} \pm 1422$ & $66,30 \mathrm{a} \pm 4,49$ & $53,07 \mathrm{~b} \pm 14,99$ & $95,32 \mathrm{a} \pm 65,63$ & $1,77 \mathrm{a} \pm 0,73$ \\
C6N & $4620 \mathrm{a} \pm 1344$ & $70,26 \mathrm{a} \pm 4,50$ & $52,77 \mathrm{~b} \pm 15,23$ & $120,59 \mathrm{a} \pm 36,62$ & $2,31 \mathrm{a} \pm 1,2$ \\
$\mathrm{~N}$ & $5075 \mathrm{a} \pm 1509$ & $53,45 \mathrm{~b} \pm 4,47$ & $49,70 \mathrm{~b} \pm 13,17$ & $96,23 \mathrm{a} \pm 63,58$ & $1,66 \mathrm{a} \pm 0,82$ \\
TA & $4833 \mathrm{a} \pm 1802$ & $54,21 \mathrm{~b} \pm 2,54$ & $38,03 \mathrm{~b} \pm 10,14$ & $92,96 \mathrm{a} \pm 39,06$ & $1,93 \mathrm{a} \pm 0,53$ \\
\hline
\end{tabular}

Les chiffres affectés de lettres identiques dans la même colonne ne sont pas significativement différents au seuil de p<0,05, selon le test de Fisher. 

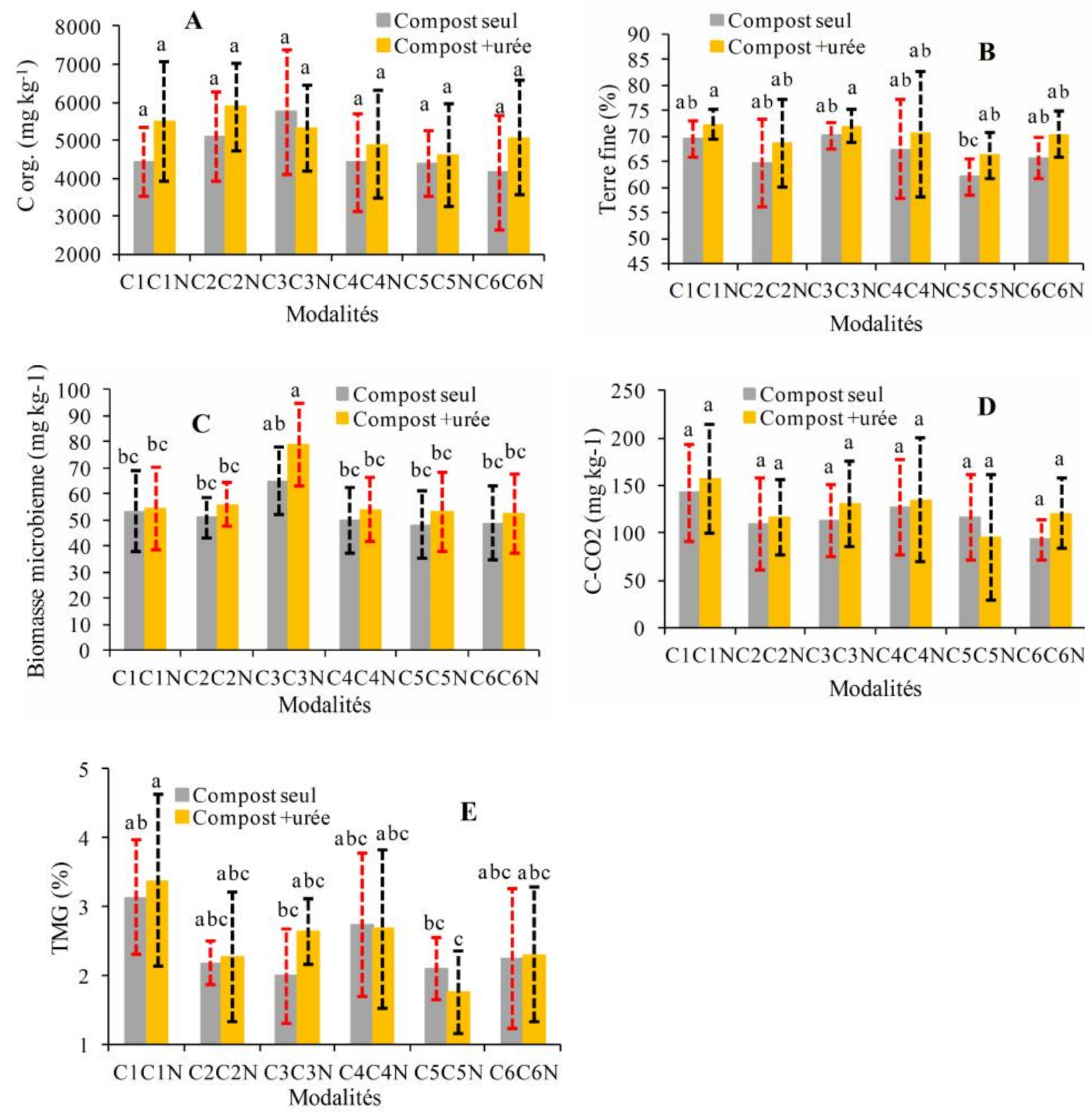

Fig.2. Comparaison des modalités C et CN : Moyennes \pm écart type. Subscripts : (A) C org., (B) Terre fine, (C) CBM, (D) Cumul de $\mathrm{CO}_{2}$ mesuré après 21 jours d'incubation des sols, (E) TMG Les paires d'histogrammes surmontés de lettres identiques indiquent une différence non significative $(p>0,05)$ des 


\subsection{Cinétique de la respiration des sols}

Les courbes d'évolution de la respiration des sols ont présenté globalement la même allure. Les dégagements de $\mathrm{C}-\mathrm{CO}_{2}$ ont été proportionnels au temps d'incubation des sols, figure 3. A B. La vitesse de minéralisation a été plus rapide au cours de la première semaine puis a diminué plus ou moins jusqu'au $21^{\mathrm{e}} \mathrm{j}$. Les sols du traitement $\mathrm{C} 1$ ont eu la plus forte vitesse de minéralisation alors que ceux du traitement TA ont montré la plus faible vitesse de minéralisation comparativement aux traitements $\mathrm{C} 2, \mathrm{C} 3, \mathrm{C} 4, \mathrm{C} 5$ et C6. La vitesse de minéralisation et la quantité de carbone minéralisées ont été plus élevées quand les composts étaient couplés à l’urée.
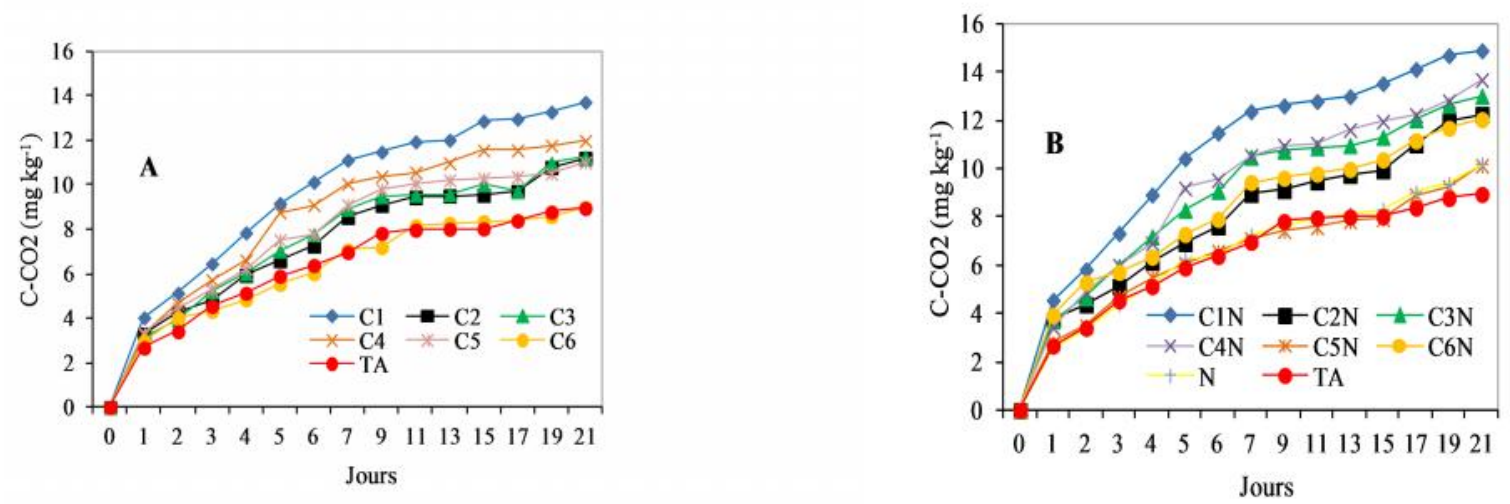

Fig.3. Cinétique du C-CO $\mathrm{CO}_{2}$ respiré sous composts seuls (A) et sous composts couplés avec l'urée (B) pendant 21 jours d'incubation des sols

\subsection{Relations entre les variables}

Le taux de minéralisation globale était lié à la respiration et à la biomasse microbienne $(r=0,720$ et 0,548$)$ et la respiration était corrélée à la biomasse microbienne $(r=0,562)$ pour les traitements à base de composts seuls (Tableau 5). Les équations des modèles de relation étaient significatives $(\mathrm{p}<0,05)$ à hautement significative $(\mathrm{P}<0,0001)$ (tableau 6). 
Tableau 5. Corrélation ( $\mathrm{r}$ de Pearson) entre les paramètres du sol pour les traitements sous composts seuls

\begin{tabular}{llllll}
\hline Variables & C-CO2 & C org & TMG & CBM & Terre fine \\
\hline C-CO2 & 1,000 & 0,310 & $\mathbf{0 , 5 6 2}$ & $\mathbf{0 , 7 2 0}$ & 0,088 \\
C org & 0,310 & 1,000 & 0,019 & $-0,377$ & 0,168 \\
TMG & $\mathbf{0 , 5 6 2}$ & 0,019 & 1,000 & $\mathbf{0 , 5 4 8}$ & 0,145 \\
CBM & $\mathbf{0 , 7 2 0}$ & $-0,377$ & $\mathbf{0 , 5 4 8}$ & 1,000 & 0,341 \\
Terre fine & 0,088 & 0,168 & 0,145 & 0,341 & 1,000 \\
\hline
\end{tabular}

Les variables dont les valeurs sont en gras indiquent une corrélation significative entre elles au seuil $\alpha=0,05$

Tableau 6. Equations des modèles de relation entre les paramètres biologiques du sol pour les traitements sous composts seuls

\begin{tabular}{lcc}
\hline Equations des modèles & Probabilités & Significativité \\
\hline TMG $(\%)=0,570+0,016 \times$ C-CO2 $(\mathrm{mg} / \mathrm{kg})$ & 0,0002 & $\mathrm{Ts}$ \\
$\mathrm{TMG}(\%)=0,488+0,036 \times \mathrm{CBM}(\mathrm{mg} / \mathrm{kg})$ & 0,010 & $\mathrm{~S}$ \\
$\mathrm{C}-\mathrm{CO} 2(\mathrm{mg} / \mathrm{kg})=25,804+1,727 \times \mathrm{CBM}(\mathrm{mg} / \mathrm{kg})$ & 0,008 & $\mathrm{~S}$ \\
\hline
\end{tabular}

Ts : Très significatif ; $S$ : Significatif

Quant aux traitements à base de composts couplés à l'urée, le taux de minéralisation globale du carbone était lié à la respiration et à la biomasse microbienne $(r=0,874$ et 0,462$)$ (Tableau 7).

Tableau 7. Corrélation ( $\mathrm{r}$ de Pearson) entre les paramètres du sol pour les traitements sous composts couplés à l'urée

\begin{tabular}{llllll}
\hline Variables & C-CO2 & C org. & TMG & CBM & Terre fine \\
\hline C-CO2 & 1,000 & 0,207 & $\mathbf{0 , 8 7 4}$ & 0,388 & 0,131 \\
C org. & 0,207 & 1,000 & $-0,249$ & $-0,183$ & 0,089 \\
TMG & $\mathbf{0 , 8 7 4}$ & $-0,249$ & 1,000 & $\mathbf{0 , 4 6 2}$ & 0,166 \\
CBM & 0,388 & $-0,183$ & $\mathbf{0 , 4 6 2}$ & 1,000 & 0,365 \\
Terre fine & 0,131 & 0,089 & 0,166 & 0,365 & 1,000 \\
\hline
\end{tabular}

Les variables dont les valeurs sont en gras indiquent une corrélation significative entre elles au seuil $\alpha=0,05$ 
Les équations des modèles ont été (i) TMG $(\%)=0,145+0,0185 \times \mathrm{C}-\mathrm{CO} 2(\mathrm{mg} / \mathrm{kg})(\mathrm{p}<0,0001)$ et (ii) TGM $(\%)=0,658+0,0306 \times \mathrm{CBM}(\mathrm{mg} / \mathrm{kg})(\mathrm{p}=0,023)$.

\section{DISCUSSION}

\subsection{Variabilité de la teneur en carbone organique}

L'application de composts a augmenté les teneurs en carbone organique du sol, teneurs influencées par la qualité des composts. Le taux élevé de carbone organique sous les composts par rapport au témoin sans fertilisation s'expliquerait par la teneur des composts en composés lignifiés qui résistent à la minéralisation rapide[48]. En effet, les composts avaient un $\mathrm{C} / \mathrm{N}$ compris entre 14 et 25, ce qui selon[31] est élevé. Par conséquent, leur apport au sol entrainerait une évolution lente de la matière organique. Il s'agit donc de composts à pouvoir amendant assez élevé [48]. Le degré d'accumulation de la matière organique dans le sol dépend en partie de la qualité des substrats organiques[49]. C'est ce qui explique les différences constatées dans les concentrations en C org d'un traitement à l'autre. Werner et Giusquiani [16-17] ont fait des constats semblables dans des études sur l'influence de divers composts sur les propriétés du sol. Mais ces auteurs ont précisé que les effets escomptés n'ont été perceptibles que dans le long terme avec de forte dose de compost.

\subsection{Variabilité du taux de terre fine}

Le taux de terre fine était élevé sous composts seuls, encore plus élevé sous composts couplés à l'urée, par contre dans le témoin, le taux était moindre. L'accroissement du taux de terre fine pourrait s'expliquer par une activité biologique plus intense sous compost, ce qui favoriserait la remontée des éléments fins des horizons plus profonds vers la surface[50]. Par ailleurs, selon[51] la matière organique des composts joue un rôle important dans la stabilisation des particules fines, réduisant ainsi les risques de leur lessivage. Selon[52], une agrégation stable procure une bonne structure au sol, une porosité plus élevée, et une protection contre l'érosion. Nos résultats sont conformes à ceux obtenus par Tejada [53] qui ont observé une augmentation de la granulométrie fine suite à l'apport de compost urbains au sol.

\subsection{Variabilité du CBM, de la respiration du sol et du TMG}

Les résultats obtenus indiquent une augmentation de la biomasse microbienne du sol sous composts comparativement au sol n'ayant reçu aucun apport de compost. Nous expliquons cette augmentation de biomasse microbienne par le fait que la majorité des populations microbiennes 
dans le sol sont des hétérotrophes[54] et donc tributaires de la disponibilité du carbone organique. Par conséquent, la modification du sol avec l'apport de compost a entrainé une augmentation de la biomasse microbienne. Par ailleurs, L'ajout de composts apporte des microorganismes vivants et représente une source nutritionnelle additionnelle pour les organismes endogènes du sol[55].

Il est admis dans la littérature que l'apport de MO dans un sol stimule l'activité microbienne générale de ce sol $[56,1,50,5]$. Les composts ayant un pouvoir absorbant maintiennent une humidité relative du sol propice à la vie microbienne [57,58]. De plus, après minéralisation des composts, la libération de nutriments améliore la croissance du sorgho qui, à son tour, fournit plus de carbone dans le sol en créant une MOS supplémentaire via les racines et leurs exsudats[54-56] ont montré qu'une variation du taux de matière organique affecte la biomasse microbienne du sol. Gaillard et al.[59] ont constaté que l'amendement du sol avec des produits organiques permet d'augmenter à la fois la respiration du sol et l'activité nitrifiante.

La cinétique de la respiration indique une vitesse et une quantité de $\mathrm{CO}_{2}$ dégagé élevées pendant la première semaine puis un ralentissement par la suite jusqu' au $21^{\mathrm{e}} \mathrm{j}$ d'incubation. Cette évolution se justifie par l'épuisement avec le temps de la quantité de carbone organique contenu dans le sol. [60], [50] et [5] ont observé une cinétique similaire lors d'incubation d'échantillons de compost ou de sols prélevés en vue d'analyser la stabilité de la matière organique.

La quantité de $\mathrm{CO}_{2}$ dégagé au bout de $21 \mathrm{j}$ d'incubation a révélé que les sols ayant reçu des composts ont une respiration plus grande que le sol témoin non amendé. Le type de composts a toutefois influencé de façon non statistiquement significative le dégagement de $\mathrm{CO}_{2}$. Le $\mathrm{C} 6$ (compost de déchets verts) avec son $\mathrm{C} / \mathrm{N}$ élevé a été le compost stimulant le moins l'activité respiratoire du sol. L'activation de la vie microbienne nécessite la présence de matière organique facilement dégradable qui lui servira de support trophique[54]. En effet, l'apport au sol de compost à $\mathrm{C} / \mathrm{N}$ élevé, du fait de son faible pouvoir de dégradation et de sa résistance aux organismes, provoque une léthargie momentanée des microorganismes qui immobilisent l'azote du sol pour leur métabolisme [58], pourtant, le dégagement du $\mathrm{CO}_{2}$ est étroitement lié à la libération d'azote minéral [61]. Il y a alors un regain d'activité microbienne suite à l'ajout d'urée à ce compost, c'est pourquoi le sol de C6N a respiré plus que celui de C6. En fait, le C6 s'est comporté comme une matière inerte, avec une respiration comparable à celle du sol témoin. C'est le C1 (compost de $60 \% \mathrm{DA}+40 \% \mathrm{DV})$ qui a induit la plus forte respiration du sol dû probablement à la forte concentration de ce produit en déchet d'abattoir (contenu des panses) qui est lui-même un substrat 
riche en microflore[62] pouvant influencer la biomasse microbienne du sol. Il est suivi du C4 $(20 \% \mathrm{DA}+40 \mathrm{DC}+40 \% \mathrm{DV})$. Cette performance du C4 serait lié non seulement à la présence du DA mais aussi au fait que DC soit un substrat riche en azote[21]. Les sols amendés aux composts couplés à l'urée ont présenté des quantités de $\mathrm{CO}_{2}$ dégagé supérieures à ceux amendés aux composts seuls. L'ajout d'urée a résolu la difficulté (faim d'azote) liée à la faible teneur des composts en azote et cela a entrainé une augmentation de l'activité microbienne au sein des sols ainsi traités se traduisant par une respiration plus forte à leur niveau. Cependant l'urée apportée seule au sol a eu un effet dépressif sur l'activité microbienne, les microorganismes n'ayant pas de support trophique et énergétique (carbone) pour optimiser la valorisation de cet azote. En effet, la biomasse microbienne est plus faible sous les traitements à base d'urée et la respiration du sol y est voisine du témoin. Les valeurs des TMG permettent de ranger les traitements par ordre de TMG décroissant comme suit $\mathrm{C} 1>\mathrm{C} 4>\mathrm{C} 6>\mathrm{C} 2>\mathrm{C} 5>\mathrm{C} 3$ pour les composts seuls et $\mathrm{C} 1 \mathrm{~N}>\mathrm{C} 4 \mathrm{~N}>$ $\mathrm{C} 3 \mathrm{~N}>\mathrm{C} 6 \mathrm{~N}>\mathrm{C} 2 \mathrm{~N}>\mathrm{C} 5 \mathrm{~N}$ pour ceux combinés avec l'urée. Le sol du C3 (30\%DA + 30\%DC + 40DV) semble être celui qui a été le plus stable avec un TMG bas, une biomasse microbienne élevée et une forte teneur en carbone organique en dehors de tout apport d'urée. Ce compost est celui qui a été produit avec le mélange le mieux équilibré comparativement aux autres composts. Les travaux de[63] et de[64] ont montré que les meilleurs composts étaient ceux produits à partir de mélanges équilibrés de substrats variés. En effet, les substrats étant de compositions chimiques différentes, plus ils sont variés et plus le compost généré renfermera des éléments chimiques variés qui lui donneront une valeur agronomique plus efficace.

Les tableaux de corrélation (cf. Tableaux 5 et 7) montrent que l'activité respiratoire est positivement et significativement liée à la biomasse microbienne. [65] ont indiqué dans leurs travaux sur l'évaluation des impacts environnementaux des techniques culturales sans labour que l'augmentation de biomasse microbienne se traduit souvent par une augmentation de la respiration. L'accroissement de la biomasse microbienne consécutif aux apports organiques a favorisé la texture fine bien que le lien n'ait pas été significatif $(0,341 \leq \mathrm{r} \leq 0,365)$ et ce, à cause de la durée limitée de l'expérimentation (3 ans) [16, 17]. En effet, ces auteurs ont rapporté qu'un minimum de quatre ans avait été requis au cours de leur expérimentation pour observer un changement significatif des paramètres du sol sous l'effet d'apports de compost. Mais, [50] ont montré que les variables telles que la biomasse microbienne, le carbone organique et la respiration sont associées dans le même sens que la texture fine du sol. La terre fine favorise l'humification et ralentit la 
minéralisation de l'humus. L'humus lié à la terre fine est protégé contre l'attaque microbienne[5, 54]. La teneur en terre fine augmente le temps de résidence (turnover) du carbone dans le sol[54]. La rhizosphère joue également un rôle important dans la dynamique et le métabolisme du carbone organique du sol[66]. Une part importante du $\mathrm{CO}_{2}$ fixé par photosynthèse dans les organes aériens des plantes est relâchée dans le sol sous forme de rhizodépositions[67]. Ces composés servent de substrats de croissance pour les micro-organismes et influencent également les caractéristiques physico-chimiques du sol environnant[67].

\section{CONCLUSION}

Les composts contribuent à l'amélioration du statut du carbone organique des lixisols par les apports de composés lignifiés, support de vie de la fraction microbienne du sol. La matière organique, la biomasse et l'activité microbienne sont plus élevées sous composts de déchets urbains comparativement à la situation sans apport de composts. La biomasse et l'activité microbiennes améliorent la texture fine. La texture fine protège la MOS contre la dégradation et maintient une bonne activité microbienne favorable à la stabilisation de la matière organique, ce qui réduit sa vitesse de minéralisation dans les conditions de température relativement élevées des régions arides. Il en découle une relative teneur élevée du sol en carbone organique. Les composts qui favorisent le mieux l'activité microbienne et l'accroissement de la teneur du sol en carbone sont ceux produits à partir de mélanges variés et équilibrés de substrats initiaux. La valorisation des composts de déchets urbains respectant les normes environnementales peut améliorer la fertilité des lixisols et contribuer à améliorer le statut du carbone organique soutenant durablement les processus microbiens.

\section{REMERCIEMENTS}

Nous remercions les projets RIPIECSA (Recherche Interdisciplinaire et Participative sur les Interactions entre les Ecosystèmes, le Climat et les Sociétés d'Afrique de l'Ouest) et LMI-IESOL (Laboratoire Mixte International-Intensification Ecologique des Sols Cultivés en Afrique de l'Ouest) pour leur appui financier lors des travaux de terrain et les analyses de laboratoires. Nous remercions Abel KADEBA doctorant au Laboratoire de Biologie et Ecologie Végétales pour son appui technique. 


\section{RÉFÉRENCES}

[1] Ganry F., Feller C., Harmand J.M., Guibert H. Management of soil organic matter in semiarid Africa for annual cropping systems. Earth and Environmental Science, 2001, 61 (1-2), 105-118.

[2] Bationo A., Kihara J., Vanlauwe B., Waswa B. and Kimetu J. Soil organic carbon dynamics, functions and management in West African agro-ecosystems. Agricultural Systems, 2007, 94, 13 25.

[3] Rajiv K.S. and Sunil H. Organic farming: producing chemical-free, nutritive and protective food for the society while also protecting the farm soil by earthworms and vermicompost reviving the dreams of Sir Charles Darwin. Agricultural Science Research Journals, 2012, 2(5), 217-239.

[4] Arrouays D., Felle, C., Jolivet C., Saby N., Andreux F., Bernoux M. et Cerri C. Estimation de stocks de carbone organique des sols à différentes échelles d'espace et de temps. Etude et Gestion des Sols, 2003, 10 (4), 347-355.

[5] Annabi M., Bahri H., Latiri K. Statut organique et respiration microbienne des sols du nord de la Tunisie. Biotechnol. Agron. Soc. Environ., 2009, 13 (3), 401-408.

[6] Bernoux, M. et Chevallier T. Le carbone dans les sols des zones sèches. Des fonctions multiples indispensables. CSFD. France, 2013, 44 p.

[7] Arrouays D., Balesdent J., Germon J.C., Jayet P.A., Soussana J.F. et Stengel P. Contribution à la lutte contre l'effet de serre : stocker du carbone dans les sols agricoles de France ? Expertise Scientifique Collective, INRA MEDD, 2002, 332 p.

[8] Boulet R. et Leprun J.C. Etude pédologique de la Haute-Volta, région Est. ORSTOM, Dakar, 1969, 319 p.

[9] Lal R. Carbon sequestration in dryland ecosystemes of west Asia and North Africa. Land Degradation Development, 2002, 13, 45-59.

[10] Zougmoré R., Ouattara K., Mando A., Ouattara B. Rôle des nutriments dans le succès des techniques de conservation des eaux et des sols (cordons pierreux, bandes enherbées, zaï et demilunes) au Burkina Faso. Sécheresse, 2004, 15 (1), 41-88.

[11] FAO. Severity of human induced soil degradation-National maps. Land and water Development Division, AGL-FAO. Rome, 2005. 
[12] Kagambèga F.W., Traoré S., Thiombiano A. et Boussim J.I. Impact de trois techniques de restauration des sols sur la survie et la croissance de trois espèces ligneuses sur les «zipellés » au Burkina Faso. Int. J. Biol. Chem. Sci., 2011, 5(3), 901-914.

[13] Roose E. Diversité des stratégies traditionnelles et modernes de conservation de l'eau et des sols. Influence du milieu physique et humain en région soudano-sahélienne d'Afrique occidentale. L'aridité une contrainte au développement, ORSTOM (Eds.), 1992, 26 p.

[14] Mando A., Zougmoré R., Zombré N.P., Hien V., 2001. Réhabilitation des sols dégradés dans les zones semi-arides de l'Afrique sub-sahélienne. In : La jachère en Afrique tropicale. C. Floret, R. Pontanier (Eds.), Vol. II., John Libbey, Paris, pp. 311-339.

[15] UICN (Union Internationale pour la Conservation de la Nature). Rapport synthèse des études de capitalisation des connaissances, pratiques, stratégies et technologies locales d'adaptation au changement climatique au Burkina Faso, Mali et Sénégal. Version finale. Projet «Intégration de l'adaptation au changement climatique dans les stratégies de réduction de la pauvreté en Afrique de l'Ouest», 2011, 22 p.

[16] Werner W., Sherer H.W. and Olfs H.W. Influence of Long-Term Application of sewagesludge and compost from garbage with sewage-sludge on soil fertility criteria. J. Agr. Crop Sci., 1988, 160, 173-179.

[17] Giusquiani P.L., Pagliai M., Gigliotti G., Businelli D. and Benetti, A. Urban waste compost: effects on physical, chemical, and biochemical soil properties. J. Environ. Qual., 1995, 24, 175182.

[18] Crecchio C., Curci M., Pizzigallo M. D. R., Ricciuti P. and Ruggiero, P. Effects of municipal solid waste compost amendments on soil enzyme activities and bacterial genetic diversity. Soil Biol. Biochem,. 2004, 36, 1595-1605.

[19] N’Dayegamiye A., Drapeau A., Laverdière M.R. Effets des apports de composts de résidus ménagers sur les rendements des cultures et certaines propriétés du sol. Agrosol., 2005, 16 (2), 135-144.

[20] United Nations. World urbanization prospects: the 2007 revision. Department of Economic and Social Affairs/Population Division. New York, 2008, 244 p.

[21] Kaboré W.T., Hien E., Zombré P., Coulibaly A., Houot S., Masse D. Valorisation de substrats organiques divers dans l'agriculture péri-urbaine de Ouagadougou (Burkina Faso) pour 
l'amendement et la fertilisation des sols : acteurs et pratiques. Biotechnol. Agron. Soc. Environ., 2011, 15 (2), 271-286.

[22] MHU (Ministère de l'Habitat et de l'Urbanisme), 2008. Schéma Directeur d'Aménagement du Grand Ouaga, Horizon 2025. Volume I : le portrait du Grand-Ouaga. AAPUI-Arcade. Ouagadougou, 2008, 216 p.

[23] INSD (Institut National de la Statique et de la Démographie). Recensement général de la population et de l'habitation de 2006. MEF, Ouagadougou, 2008, 52 p.

[24] Fontès J. et Guinko S. Carte de la végétation et de l'occupation du sol du Burkina Faso. Note explicative. Projet Campus 88313 101. Ouagadougou, 1995, 67p.

[25] BUNASOLS. Etude morpho-pédologique de la province d'Oubritenga et du Kourwéogo (échelle 1/50 000). Rapport technique. MA, Ouagadougou, 1998, 42 p.

[26] WRB (World Reference Base for soil resources). World reference base for soil resources. A framework for international classification, correlation and communication. IUSS-ISRIC-FAO. Rome, 2006, 128 p.

[27] CPCS (Convention de Pédologie et de Classification des Sols). Classification des sols. ENSA. Grignon, 1967, 77 p.

[28] Richer de Forges A.C. Lost in the triangular diagrams of soil texture. Pedometron, 2010, 1417.

[29] BUNASOLS (Bureau National des Sols). Manuel pour l'évaluation des terres. Document technique $n^{\circ} 6$, Ouagadougou, 1990, $181 \mathrm{p}$.

[30] AFNOR (Agence Française de Normalisation). Normes Françaises NF U44-051. Amendements organiques : dénominations, spécifications et marquage, Paris, 2006, 28 p.

[31] Pouech P. La valeur agronomique des composts : Synthèse bibliographique. ADAESOAPESA, France, 2006, 42 p.

[32] INERA (Institut de l'Environnement et de Recherches Agricoles). Fiche technique du sorgho CEF 382/2-1-1 (sariaso 11). Création INERA/CIRAD, Station de Recherche de Saria, Burkina Faso, 1998, 2 p.

[33] Lamaze T., Khamis S., Foyer C., Farineau J., Valadier M.H., Morot-Gaudty J.F. Effet d'une limitation en $\mathrm{N}$ sur la photosynthèse chez le maïs. In: Physiologie et production du maïs, INRA (Eds), Paris, 1990, 113-121. 
[34] Chopart, J.L. Systèmes racinaires des cultures annuelles tropicales : effets du travail du sol sur les racines. In : Le Travail du Sol pour une Agriculture Durable, Hoogmoed, W.B., Klaij, M.C. (Eds.), Document FAO, Rome, 1994, pp 27-34.

[35] Aït-Houssa, A., Benbella, M., Badraoui, M. Eléments d'aide au raisonnement de l'échantillonnage du sol en parcelle agricole. Rev. HTE, 2004, 129, 20-29.

[36] CIRAD (Centre de Coopération Internationale en Recherche Agronomique pour le Développement). Catalogue des analyses courantes, code 112 : Azote et Carbone totaux (combustion). CIRAD, France, 2004, 28 p.

[37] Blanchart E., Bernoux M. Déterminants des stocks de carbone des sols des petites Antilles (Martinique, Guadeloupe). Alternatives de séquestration du carbone et spatialisation des stocks actuels et simules. Subvention 01105, MEDD, 2005, 104 p.

[38] Jenkinson D.S. and Powlson D.S. The effects of biocidal treatments on metabolism in soil. A method for measuring soil biomass. Soil Biol. Biochem., 1976, 8, 209-213.

[39] Kaiser E.A., Mueller T., Joergensen R.G., Insam H. et Heinemeyer. Evaluation of methods to estimate the soil microbial biomass and the relationship with soil texture and organic matter. Soil Biology Biochemistry, 1992, 24, 675-683.

[40] Wang W.J., Dalal R.C., Moody P.W. et Smith C.J. Relationship of soil respiration to microbial biomass, substrate availability and clay content. Soil Biology Biochemistry, 2003, 35, 273-284.

[41] Chaussod R., Nicolardot B. and Catroux G. Mesure en routine de la biomasse microbienne des sols par la méthode de fumigation au chloroforme. Sci. Sol, 1986, 2, 201-211.

[42] Dommergues Y. La notion de coefficient de minéralisation du carbone dans les sols. Agron. Trop., 1960, 15 (1), 55-60.

[43] ITAB (Institut Technique de l'Agriculture Biologique). Activités biologiques et fertilité des sols: Intérêts et limites des méthodes analytiques disponibles, France, 2002, 25 p.

[44] Traoré S. Les formations à Acacia de l'Est du Burkina Faso: typologie en relation avec les facteurs pédoclimatiques, prédictions spatiales et fonction sur la dynamique du carbone et de l'azote. Thèse de doctorat, Univ. Ouagadougou, 2008, 176 p.

[45] Morel J.L., Guckert A., Nicolardot B., Benistant D., Catroux G., Germon J. C. Etude de l'évolution des caractéristiques physico-chimiques et de la stabilité biologique des ordures ménagères au cours du compostage. Agronomie, 1986, 6 (8), 693-701. 
[46] Assa A., Sedogo M.P., Sawadogo L., Pallo F.J.P. et Sawadogo N. Statut de la matière organique des sols dans la zone sud-soudanienne au Burkina Faso. Biotechnol. Agron. Soc. Environ., 2008, 12 (3), 291-301.

[47] Addinsoft. XLSTAT-Pro 7.5.2. User's manual, 2004, 230 p.

[48] Francou C. Stabilisation de la matière organique au cours du compostage de déchets urbains: Influence de la nature des déchets et du procédé de compostage - Recherche d'indicateurs pertinents. Thèse d'INAPG, Grignon, 2003, 388 p.

[49] Sagar S., McIntosh P.D., Hedley C.B. and Knicker H. Changes in soil microbial biomass, metabolic quotient, and organic matter turnover under Hieracium (H pilosella L.) Biology and Fertility of Soils, 1999, 30, 232-238.

[50] Traoré S., Thiombiano L., Millogo J.R. and Guinko S. Carbon and nitrogen enhancement in Cambisols and Vertisols by Acacia spp. in eastern Burkina Faso: Relation to soil respiration and microbial biomass. Applied Soil Ecology, 2007, 35, 660-669.

[51] Watts C.W., Whalley W.R., Brookes P.C., Devonshire B.J and Whitmore A.P. Biological and physical processes that mediate micro-aggregation of clays. Soil Science, 2005, 170, 573-583.

[52] Le Bissonnais Y., Cros-Cayot S., Gascuel-odoux C. Topographic dependence of aggregate stability, overland flow and sediment transport. Agronomie, 2002, 22, 489-501.

[53] Tejada M. et Gonzalez J.L. Influence of two organic amendments on the soil physical properties, soil losses, sediments and runoff water quality. Geoderma, 2008, 145 (3-4), 325-334.

[54] Huber G., Schaub C. La fertilité des sols : l'importance de la matière organique. Agriculture et Territoire. Chambre d'Agriculture, Bas-Rhin, 2011, 46 p.

[55] Chalhoub M. Effet de l'apport de composts sur la dynamique hydrique du sol, la disponibilité de l'azote pour la culture et le lessivage du nitrate : cas d'un sol limoneux cultivé du Bassin parisien. Thèse, Université de Paris-Sud 11, 2010, 258 p.

[56] Powlsen D.S., Brookes P.C. and Christensen B.T. Measurement in soil microbial biomass provides an early indication of changes in the total organic matter due to straw corporation. Soil Biology Biochemistry, 1987, 19, 159-164.

[57] Salducci X. Diagnostic de la fertilité biologique des sols et gestion de la matière organique : concepts - méthodes - applications. Société Celesta-lab, France, 2005, 46 p. 
[58] Landry C., Boivin C., Belzile L., Mainguy J. Essais sur l'apport de différents types de matières organiques appliquées en bande à l'implantation d'un verger de cassis. IRDA, Canada, 2013, 50 p.

[59] Gaillard V., Chenu C., Recous S., Richard G. Carbon, nitrogen and microbial gradients induced by plant residues decomposing in soil. Eur. J. Soil Sci., 1999, 50, 567-578.

[60] Houot S., Francou C., Vergé-Leviel C., Michelin J., Bourgeois S., Linères M., Morel P., Parnaudeau V., Le Bissonnais Y., Dignac M.F., Dumat C., Cheiab A., Poitrenaud M. Valeur agronomique et impacts environnementaux de composts d'origine urbaine : variation avec la nature du compost. Dossier de l'environnement de l'INRA, 2003, 25, 107-124.

[61] Delphin J.E. Utilisation de cinétiques du premier ordre en vue de caractériser la minéralisation de quelques sols agricoles. Agronomie, 1988, 8 (4), 289-294.

[62] Diallo A. Problématique de la gestion des déchets animaux, cas des abattoirs de Dakar. Thèse, Univ. Cheik Anta Diop, EISMV, 1999, 90 p.

[63] Znaïdi I.E.A. Etude et évaluation du compostage de différents types de matières organiques et des effets des jus de composts biologiques sur les maladies des plantes. Master de CIHEAMCTAB, Tunisie, 2002, $104 \mathrm{p}$.

[64] Hugh M. Introduction au compostage agricole. Fiche technique. MAA, Ontario, 2005, 12 p.

[65] Labreuche J., Le Souder C., Castillon P., Ouvry J.F., Real B., Germon J.C., De Tourdonnet S. Evaluation des impacts environnementaux des Techniques Culturales Sans Labour en France. ADEME-ARVALIS, France, 2007, 400 p.

[66] Marilley L., Vogt G., Aragno M. Diversité bactérienne du sol et de la rhizosphère et effet d'une augmentation en CO2 atmosphérique. Poster, 1994.

[67] Lynch J.M. Introduction: some consequences of microbial rhizosphere competence for plant and soil. In: J. M. Lynch (Eds). The Rhizosphere. John Wiley \& Sons Ltd, Essex, 1990, pp 1-10.

\section{RESUME}

La minéralisation est une cause de déstockage du carbone du sol. Cette étude s'est intéressée aux mesures de l'activité microbienne en lien avec des apports de composts dans un essai de sorgho durant trois ans. Les composts ont été apportés seuls ou combinés à l'urée. La teneur en carbone organique, la biomasse microbienne, la respirométrie et le taux de minéralisation globale du carbone ont été mesurées sur des échantillons de sol en fin d'expérimentation. Les variations des 
paramètres par rapport au témoin fluctuaient entre -1 et $+71 \%$ sous composts seuls et entre 0 et $+107 \%$ sous composts couplés à l'urée. Sous urée pure, les paramètres ont varié de $-14 \%$ à $+31 \%$. Les composts performants semblaient être ceux produits avec des substrats équitablement variés. La valorisation de composts écologiques peut améliorer le statut du carbone du sol soutenant durablement les processus microbiologiques.

Mots-clés : Agro-systèmes, carbone, respirométrie, Burkina Faso.

How to cite this article:

Bambara D. Thiombiano A. Hien V. A lixisol microbial activity under urban wastes composts effect in Donsin, Burkina Faso. J Fundam Appl Sci. 2014, 6(2), 238-262. 